\title{
A Simple Micropreparative Gel Electrophoresis Technique For Purification of Nanoscale Materials
}

\section{Sayyed Hashem Sajjadi}

École Polytechnique Fédérale de Lausanne (EPFL)

\section{Shang-Jung Wu}

École Polytechnique Fédérale de Lausanne (EPFL)

\section{Vitalijs Zubkovs}

École Polytechnique Fédérale de Lausanne (EPFL)

\section{Hossein Ahmadzadeh}

Ferdowsi University of Mashhad

\section{Elaheh K. Goharshadi}

Ferdowsi University of Mashhad

Ardemis A. Boghossian ( $\square$ ardemis.boghossian@epfl.ch )

École Polytechnique Fédérale de Lausanne (EPFL)

\section{Research Article}

Keywords: Preparative electrophoresis, Protein, DNA, Protein-DNA conjugate, Nanoparticles, Graphene quantum dots, Chromatography.

Posted Date: December 14th, 2021

DOI: https://doi.org/10.21203/rs.3.rs-1162722/v1

License: (c) (i) This work is licensed under a Creative Commons Attribution 4.0 International License.

Read Full License 


\section{Abstract}

Many biochemical, biomedical, and material applications hinge on the ability to effectively separate and purify nanoscale materials. Though this need is largely addressed with biological macromolecules using a variety of chromatographic and electrophoretic purification techniques, such techniques are usually laborious, time-consuming, and often require complex and costly instalments that are inaccessible to most laboratories. Synthetic nanoparticles face similar purification challenges, often relying on techniques that are material-specific. In this work, we introduce a versatile micro-preparative (MP) method based on polyacrylamide gel electrophoresis (PAGE) to purify biological samples containing proteins, nucleic acids, and complex bioconjugates, as well as synthetic nanoparticles based on graphene quantum dots (GQDs). Using a conventional vertical slab PAGE, we demonstrate the extraction of purified DNA, proteins, and DNA-protein bioconjugates from their respective mixtures using MP-PAGE. We apply this system to recover DNA from a ladder mixture with yields of up to $90 \%$, compared to the $58 \%$ yield obtained using specialized commercial devices. We also demonstrate the purification of folded enhanced yellow fluorescence protein (EYFP) from crude cell extract with $90 \%$ purity, comparable to purities achieved using a two-step size exclusion and immobilized metal-ion affinity chromatography purification procedure. Moreover, we demonstrate the successful isolation of an EYFP-DNA bioconjugate that otherwise could not be processed using the two-step chromatography procedure. Finally, the technique was further extended to demonstrate size-dependent separation of a commercial mixture of GQDs into three different fractions with distinct optical properties. MP-PAGE thus offers a rapid and versatile means of purifying biological and synthetic nanomaterials without the need for specialized equipment.

\section{Introduction}

The separation and purification of biological macromolecules are crucial not only for bioanalytics but also for advancements in biotechnology [1]. Macromolecules, in particular proteins and nucleic acids, can be isolated using a slew of techniques including high-speed centrifugation, membrane-based ultrafiltration, precipitation, electrophoresis, and chromatography [2-8]. These techniques differ in aspects such as scalability, throughput, yield, purity, precision, laboratory accessibility, and procedural complexity. Their advantages are used to identify the technique or combination of techniques that are most suitable for isolating a particular macromolecule under specific conditions for a given application. In fact, protein purification remains an evolving challenge that largely hinges on the researcher's ability to piece together a suitable protocol based on existing techniques and optimization procedures. The diversity of these complementary techniques is therefore key to versatile protein isolation.

While flow chromatography systems especially benefit from relatively high throughput and scalability [5, $9,10]$, several analytical applications such as sequencing and protein crystallography require only a small amount of purified protein. For such applications, techniques such as polyacrylamide gel electrophoresis (PAGE) are used to separate biomolecules according to their size and net electric charge $[11,12]$. The pore size in polyacrylamide gel (PAG) can be precisely controlled to separate biomolecules of a particular size distribution with no need to use specialized equipment or columns for different size ranges, as performed 
in chromatography systems. In fact, PAGE serves as the gold standard for separating DNA with a single base pair resolution $[13,14]$. Due to its high resolution, low cost, and facile and rapid separation, PAGE is considered a standard and widespread analytical separation technique that can be found in most biological laboratories. In addition to DNA, this technique is especially useful for separating proteins either in their native or denatured forms [11].

While PAGE is typically used for analytical protein separation and identification, several studies have explored its use as a preparative procedure for purified protein isolation [15-24]. Large-scale (milligrams to grams of protein) electrophoresis can be achieved using preparative electrophoresis columns. However, the heat generated from this setup often leads to band broadening and protein denaturation [11]. For this reason, such setups require costly and specialized preparative gel electrophoresis equipment that is coupled with a cooling system. These separations are usually followed by post-elution methods for extracting the proteins from PAG after separation [25]. In addition, depending on the post-elution method, these extractions may require specialized setups, and the two-step separation and extraction procedure may alter protein activity and/or folding. Hao et al. [18] developed a novel microscale preparative electrophoresis system for protein separation. Although they confirmed high resolution and sufficient recovery of proteins based on gel electrophoresis, distinct separation and elution apparatuses was required. In some cases, specialized membranes are required for analysis. Fadouloglou [20], for instance, used a membrane in the electrophoresis setup to separate DNA. Although the custom-made configuration boasts advantages of its own, this customization requires a dedicated effort for constructing and troubleshooting the setup, which often limits the accessibility of the technology to specialized laboratories.

Despite the variety of preparative gel electrophoresis settings, previous techniques have been applied mainly to either DNA or proteins. Losses associated with complex and multi-step extractions often preclude the use of these techniques for the effective purification of dilute and low-yield samples, such as certain DNA-protein bioconjugates. While the Crush-and-Soak method represents the primary gel electrophoresis approach for bioconjugate purification [26], significant losses in the extraction step limit its use to concentrated samples. This method therefore prohibits the purification of a range of bioconjugate samples, including the dilute samples used in the study herein. Consequently, the purification of such dilute complexes has traditionally been limited to either chromatography techniques or multi-step approaches based on biomolecule tagging [27]. Zhou et al. [28] purified DNA-protein conjugates through a complex, multi-step procedure requiring DNA modification and magnetic bead binding and release steps, resulting in the extraction of a modified conjugate containing a desthiobiotinylated tag. Yang et al. [29] had to rely on a sophisticated membrane-based approach for biomolecule isolation. Although this work presents a novel approach for separation, the large amount of impurities resulting from this method reveal ongoing challenges in biomolecule purification.

Herein, we present a single-step micropreparative (MP)-PAGE technique for double-stranded (ds) DNA and protein purification. The tri-layer slab gel arrangement, shown in Fig. 1, utilizes a standard vertical electrophoresis setup available in most bioanalysis laboratories. The DNA extraction was compared to 
that achieved with the Crush-and-Soak method. In addition, the purities of the MP-PAGE protein samples were further analyzed and compared to those achieved with size exclusion chromatography (SEC) and immobilized metal ion affinity chromatography (IMAC) using recombinant enhanced yellow fluorescent protein (EYFP) as a model protein. The diminished losses and simplicity of this arrangement allowed us to apply MP-PAGE to a dilute and low-yield DNA-protein bioconjugate solution. This latter demonstrationn allo wously been unsiccessfully oppor allows us to demonstrate a gel electrophoresis approach that can be used to purify dilute, low-yield bioconjugate samples that are otherwise intractable using existing gel electrophoresis techniques. Purification of low amount samples is especially useful for synthetic nanomaterials. To this end, this technique was extended to separate synthetic graphene quantum dots (GQDs) from a commercial mixture to obtain purified fractions with optical properties distinct from the stock.

\section{Materials And Methods}

\section{Preparation of EYFP enriched protein extract}

Competent E. coli BL21 cells harboring the recombinant plasmid encoding EYFP were cultured in 2xYT medium supplemented with carbenicillin antibiotics. The cell culture was harvested and lysed for protein extraction. The insoluble materials were pelleted by centrifugation, and the supernatant (cell extract) was filtered through a $0.2 \mu \mathrm{m}$ porous sterile filter and stored at $4{ }^{\circ} \mathrm{C}$.

\section{EYFP purification using chromatography techniques: IMAC and SEC}

IMAC purification was performed using a His-Trap HP $1 \mathrm{ml}$ (GE Healthcare) on an ÄKTA START protein purification system (GE Healthcare). The sample was loaded in a buffer containing $20 \mathrm{mM}$ phosphate buffer saline (PBS, pH 7.4), $500 \mathrm{mM} \mathrm{NaCl}$, and $20 \mathrm{mM}$ imidazole and then eluted in $20 \mathrm{mM}$ PBS containing $500 \mathrm{mM} \mathrm{NaCl}$, and $500 \mathrm{mM}$ imidazole. $15.0 \mathrm{ml}$ of the cell extract was loaded into the column and the chromatography was carried out at a flow rate of $1.0 \mathrm{ml} / \mathrm{min}$ for all steps (loading, washing, and His-tagged protein elution).

The SEC purification was achieved using a HiPrep 16/60 Sephacryl S-300 high resolution column (GE Healthcare) on the same ÄKTA system. The elution buffer contained $10 \mathrm{mM}$ PBS ( $\mathrm{pH}=7.4)$, and $140 \mathrm{mM}$ $\mathrm{NaCl} .3 .0 \mathrm{ml}$ of the sample was loaded into the column. The chromatography was carried out for $4 \mathrm{~h}$ with a flow rate of $0.5 \mathrm{ml} / \mathrm{min}$. The sample mixture was separated and eluted into more than $40 \mathrm{fractions}$. The eluted fractions containing purified protein samples were concentrated using a $10 \mathrm{kDa}$ Amicon centrifugal filter (Merck Millipore) and the elution buffer suspending the purified protein was replaced with EDTA (10 mM)-PBS (1x) buffer $(\mathrm{pH}=7.0)$ for long-term storage. 


\section{EYFP conjugation to ssDNA}

sSDNA-EYFP bioconjugation was performed as described previously [30]. The purified EYFP was reduced using $10 \mathrm{mM}$ Tris(2-carboxyethyl)phosphine hydrochloride (TCEP, ABCR) in EDTA-PBS buffer for $1 \mathrm{~h}$ while shaking at $500 \mathrm{rpm}$ in an Eppendorf thermomixer at $5^{\circ} \mathrm{C}$. The product was purified using a PD midiTrap G-25 desalting column (GE Healthcare) eluted with the same buffer. Freshly prepared $20 \mathrm{mM}$ dibenzocyclooctyne-maleimide (DBCO, Tokyo Chemical Industry) in dimethyl sulfoxide was mixed with reduced EYFP $(0.05 \mathrm{mM})$. The sample was shaken overnight at $5{ }^{\circ} \mathrm{C}$, and the non-reacted DBCO was subsequently removed using a PD midiTrap G-25 desalting column. EYFP-DBCO was mixed with $5^{\prime}-\mathrm{N}_{3}{ }^{-}$

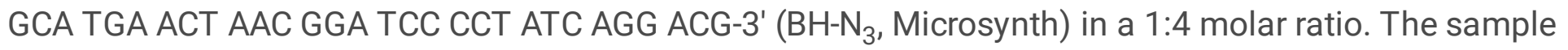
was left to react at room temperature accompanied by shaking for $4 \mathrm{~h}$. A mixture of EYFP (without DBCO) and $\mathrm{BH}$ (without azide group) was incubated in the same way as the control.

\section{MP-PAGE separation of DNA and protein samples}

The concentration of the PAG could be varied between 4 and $20 \%$, depending on the size of the biomolecule of interest. The gel contained a $50 \%$ glycerol layer in running buffer for collecting the target band, followed by a supporting gel layer in running buffer (Fig. 1). After sample loading, the electrophoresis was run until the band of the target molecule reached the glycerol layer. Prior to its elution into the glycerol layer, this layer and the supporting gel layer were replaced with new layers to remove undesired species with higher mobilities than the biomolecule of interest. The electrophoresis was reapplied until the band of interest was eluted into a new glycerol layer. The eluted sample was then recovered with a syringe and further concentrated using centrifugal filters for protein and protein-DNA conjugate samples and ethanol precipitation for DNA sample.

An acrylamide/bisacrylamide stock solution of 40\% (19:1) was used to prepare PAGs in both preparative and analytical DNA-PAGE experiments while a 30\% (37.5:1) solution was used for the protein-PAGE experiments. Both stock solutions were purchased from Carl Roth.

\section{DNA separation}

A ds-DNA ladder (HyperLadder 25-500 bp, Bioline) was pre-stained with Sybr-Gold (SG, Invitrogen) and incubated in sample buffer ( $50 \%$ glycerol with $2 x$ tris-boric acid-EDTA, TBE) for 30 min before injection into the gel. The pre-staining allowed us to track the DNA bands during electrophoresis. The preparative separation of DNA was performed on a MP-PAGE gel consisting of a $3 \mathrm{~cm} \mathrm{12 \%} \mathrm{resolving} \mathrm{PAG} \mathrm{and} 2 \mathrm{~cm}$ $50 \%$ glycerol layer with 2xTBE buffer. After extraction of the glycerol layer, the separated DNA sample was concentrated by ethanol precipitation, which also removed the tracking dye. For comparison, the same bands were also separated using a conventional post-elution extraction procedure known as Crush-andSoak [31]. For this procedure, the DNA mixture was run in a normal native PAG with the same 
concentration as the resolving gel in the MP-PAGE setup. The bands of interest were cut after electrophoresis using a scalpel, excised with a needle into small pieces, and soaked in buffer (1xTBE). The sample was incubated overnight, and the DNA that diffused out of the gel was concentrated by ethanol precipitation. The purity and recovery yield of the separated samples were compared using analytical electrophoresis on a $12 \%$ resolving PAG. To estimate the recovery yield, the corresponding volumes of the concentrated sample (taking into account the dilution factor for the recovered sample during ethanol precipitation) and original mixture were run on an analytical gel. The gel profiles were digitized using Image $\mathrm{J}$ software. The area of each peak was integrated and divided by the area of the corresponding peaks from the original mixture.

\section{Protein separation}

MP-PAGE was used to purify EYFP from the crude cell extract. The preparative separation of EYFP was performed on a gel consisting of a $3 \mathrm{~cm} 12 \%$ native resolving PAG and $2 \mathrm{~cm} 50 \%$ glycerol layer with trisglycine buffer. $300 \mu$ of crude extract, containing $~ 1.6 \mathrm{mg}$ of EYFP (according to its characteristic absorption peak at $514 \mathrm{~nm}$ with an extinction coefficient, , of $83400 \mathrm{M}^{-1} \mathrm{~cm}^{-1}$ or $2.98 \mathrm{ml} \mathrm{mg}^{-1} \mathrm{~cm}^{-1}$ ) [32] was loaded onto the MP-PAGE gel. The sample was run at $150 \mathrm{~V}$ until the fluorescent band migrated into the glycerol layer.

The purity of the protein samples extracted either via MP-PAGE or chromatography was compared using reducing sodium dodecyl sulphate (SDS)-PAGE in tris-glycine-SDS (TGS) buffer. $15 \mu$ l of each sample containing $1.0 \mu \mathrm{g}$ of EYFP (based on $A_{514} \mathrm{~nm}$ ) in reducing sample buffer (containing dithiothreitol) was loaded onto a gel consisting of $4 \%$ stacking and $12 \%$ resolving PAGs. After $1 \mathrm{~h}$ of electrophoresis at 150 $\mathrm{V}$, the gel was stained by Coomassie brilliant blue (CB).

The protein concentration of the extracted samples was determined from UV-visible spectra recorded using a NanoDrop 2000 (Thermo Scientific). $1.3 \mu \mathrm{l}$ of each sample was loaded onto the device, and the spectrum was recorded in the $250-600 \mathrm{~nm}$ range. Total protein concentration was determined using a bicinchoninic acid (BCA) assay from the Pierce ${ }^{\mathrm{TM}}$ BCA Protein Assay Kit (Thermo Scientific). The fluorescence spectra of the EYFP samples were measured with a Varioskan LUX Multimode Microplate Reader (Thermo Scientific).

\section{DNA-Protein conjugate purification}

The conjugate sample was purified using the same gel as described in the previous section (Protein separation). To assess the purity of the MP-PAGE conjugate, we performed reducing SDS-PAGE for $1 \mathrm{~h}$ at $150 \mathrm{~V}$ in TGS buffer (followed by CB staining) and urea-PAGE for $45 \mathrm{~min}$ at $200 \mathrm{~V}$ in TBE buffer (followed by SG staining) for protein and DNA detection, respectively. 


\section{GQD purification}

The commercial GQDs (Sigma-Aldrich, diameter $<5 \mathrm{~nm}$ ) were loaded in a three-layer gel consisting of a $20 \%$ PA separating gel. The gel was run at $150 \mathrm{~V}$ until the three most prominent bands were separated ( 2 h). Tris-glycine (TG) solution ( $50 \mathrm{mM} \mathrm{TG}, \mathrm{pH}$ 8.3) was used as the running buffer. The separated bands were characterized using UV-visible spectroscopy (Shimadzu 3600 Plus UV-Vis-NIR spectrophotometer) and fluorescence spectroscopy (Varioskan).

\section{Results And Discussion Separation of ds-DNA}

We applied the MP-PAGE setup shown in Fig. 1 to separate and extract individual dsDNA bands from a ladder mixture. In this setup, the desired bands were eluted and collected from the glycerol layer. Compared to buffered aqueous solutions used in specialized electrophoresis flow systems [20, 25], the higher viscosity of the glycerol layer sufficiently impedes band mobility, allowing distinct DNA bands to remain separated in the glycerol layer. In addition, the denser glycerol layer shows limited diffusion into the PAG pre-solution, allowing the pre-solution to be solidified in the presence of the neighboring glycerol layer.

This arrangement was used to separate a 25-bp band from a 12-strand ds-DNA mixture (Fig. 2A). As shown in Fig. 2A, we were also able to sufficiently separate 50 and 75-bp DNA strands using the same gel composition and operating voltage conditions, demonstrating the versatility of this technique in separating different DNA sizes from within a mixture. The calculated recovery yields were 90,80 , and 77 $\%$ for the 25, 50, and 75-bp DNA strands, respectively (Fig. 2B). The gradual decrease in the yields with increasing DNA length is attributed to the resolving gel conditions being optimized to favor separation of the $25 \mathrm{bp}$ strand. The same DNA strands were also separated using the traditional Crush-and-Soak method with calculated recovery yields of 58,54 , and $24 \%$ for the 25,50 , and 75 -bp DNA bands, respectively. Compared to MP-PAGE, the Crush-and-Soak method showed lower extraction yields and an even greater decrease in the extraction of larger DNA strands under the tested conditions. This difference in DNA extraction is largely attributed to the diffusion-limited separation of the DNA from the solid gel matrix in the Crush-and-Soak extraction [33].

\section{Protein purification: separation of EYFP from E. coli extract}

The MP-PAGE was also used to recover over-expressed EYFP from recombinant E. coli. Fig. 3A shows the analytical reducing SDS-PAGE gel of the crude bacterial extract enriched with the recombinant EYFP, accompanied by a number of contaminating proteins ranging between 10 and $100 \mathrm{kDa}$. The EYFP is represented by a double band at around the expected size of $27 \mathrm{kDa}$. This double band is attributed to the cleavage of a signaling sequence for some of the proteins [34]. The proteins contained by the bands were 
extracted and purified using MP-PAGE, SEC, IMAC, as well as a double-extraction method based on IMAC extraction followed by SEC (chromatograms and corresponding CB stained gels shown in Figs. S1 and S2). Compared to the MP-PAGE sample, which only showed sparse, faint contaminating protein bands, the SEC sample contained a greater amount of both smaller and larger protein impurities under the tested conditions. The impurities were particularly pronounced for sizes neighboring the desired $\sim 27 \mathrm{kDa}$ band. In contrast, the IMAC extraction yielded a significantly reduced level of impurities with a pronounced contaminating protein band at around $10 \mathrm{kDa}$. These impurities are attributed to non-specific binding of histidine- or arginine-rich proteins that can intrinsically bind to the nickel column. Compared to SEC and IMAC alone, the double-extraction IMAC+SEC method yielded the least amount of contamination, showing only faint impurity protein bands. Based on the gel analysis, the sample purity was comparable to that achieved with MP-PAGE, except for a faint impurity band at $40 \mathrm{kDa}$ that was more pronounced in the MP-PAGE sample. As such, the samples purified from the IMAC+SEC method were used in the subsequent analysis to compare the integrity of the proteins isolated with MP-PAGE.

The purity and integrity of the samples were further analyzed using absorption and fluorescence spectroscopies. All extracted samples, except the desaturated EYFP, showed an absorption peak at 514 nm (Fig. 3B). Furthermore, Fig. 3C shows the fluorescence of EYFP with its characteristic peak at around $533 \mathrm{~nm}$ upon $490 \mathrm{~nm}$ excitation. Both absorption and fluorescence peaks were diminished when the samples were heated to $95^{\circ} \mathrm{C}$ for $5 \mathrm{~min}$. Since this treatment denatures EYFP, the diminished absorption and fluorescence peaks verify that the detected optical signatures correspond to properly folded protein. The absorption spectra were also used to calculate sample purity. Assuming a relatively pure protein solution from the IMAC-SEC sample, we calculated an effective EYFP extinction coefficient of $1.23 \mathrm{ml}$ $\mathrm{mg}^{-1} \mathrm{~cm}^{-1}$ based on total protein concentration (as determined by the BCA assay) of the IMAC-SEC sample and the optical absorption () measured at $280 \mathrm{~nm}$, which corresponds to the non-specific absorption peak of proteins containing aromatic residues. Total native EYFP protein concentration was calculated based on its specific absorption peak at $514 \mathrm{~nm}$. By comparing the calculated protein concentration at $514 \mathrm{~nm}$ with the effective concentration at $280 \mathrm{~nm}$, the native-EYFP purity () in the different samples was calculated using the following equation:

$\% P=\frac{\left[A_{\text {sample }, 514} / \varepsilon_{E Y F P, 514}\right]}{\left[A_{\text {sample }, 280} / \varepsilon_{E Y F P, 280}\right]}$

Based on this calculation, the protein purity was found to be $16,58,94$, and $90 \%$ for SEC, IMAC, IMAC+SEC, and MP-PAGE, respectively.

\section{Purification of DNA-protein conjugate}

Bioconjugates are used in a variety of applications ranging from nanofabrication to target recognition, signal amplification, catalysis and chemical modification, and vaccines and immune modulation $[28,35$, 
36]. Several approaches have been developed to conjugate proteins and DNA [37, 38]. However, the conjugation product often requires extensive treatment to remove unreacted precursors. Methods such as the Crush-and-Soak, which was used for the DNA recovery described above, result in poor recovery yields $[26,39]$. Alternative methods such as the biotin displacement strategy developed by Zhou et al. [28], ion-exchange chromatography [40], and SEC [41] can achieve higher yields, though the extracted conjugates are often accompanied by sample impurities such as unreacted reagents as well as the need for specialized equipment and columns.

In our study, we attempted to purify a sSDNA-EYFP bioconjugate synthesized via azide-DBCO click chemistry. Specifically, ssDNA-azide was mixed with DBCO-EYFP to form a covalently linked ssDNA-EYFP based on stable triazole formation. The limited amount of sample could not be processed using SEC due to sample loss from significant dilution and the inability of SEC to discern unreacted proteins from their conjugates. However, the limited sample amount was sufficient for MP-PAGE purification. The successful conjugation of the ssDNA-EYFP was confirmed with urea-PAGE, which verified the formation of a new conjugate band following the reaction (Fig. 4A). The conjugated sample showed decreased electrophoretic mobility compared to the non-conjugated EYFP due to the appended negatively charged oligonucleotide [42]. Conjugation was also confirmed with SDS-PAGE (Fig. 4B) with a $~ 9 \mathrm{kDa}$ increase in molecular weight corresponding to the attachment of one oligonucleotide per EYFP. Since the electrophoretic mobility of DNA (Fig. 4A) and protein (Fig. 4B) did not change in the absence of azide and DBCO, the new bands appearing in the samples originate from the covalent conjugation of EYFP-DNA. The presence of unreacted DNA and protein is confirmed by the SDS-PAGE even after filtration through a $30 \mathrm{kDa}$ centrifugal membrane. In contrast, bioconjugated sample purified through MP-PAGE shows complete isolation of the conjugate from the unreacted compounds

\section{Purification of GQDs}

The ability to fractionate low amount and dilute samples is especially useful for processing costly or difficult-to-scale synthetic nanomaterials. In particular, nanomaterials such as GQDs are available as commercial mixtures with photoluminescence properties varying with size, shape, and functionality, as well as type and extent of defects $[43,44]$. Compared to conventional reporters such as fluorescent proteins, inorganic quantum dots, and organic dyes [45], GQDs benefit from both enhanced photostability and tunable photoluminescence wavelengths that motivate their use in various optoelectronic applications [46-51]. The ability to readily fractionate GQDs of different wavelengths, therefore, facilitates the development of optimizing, high-efficiency optical devices as well as multi-modal optical technologies.

MP-PAGE was thus extended to demonstrate the separation of GQDs. Since the GQDs contain several functional groups TG buffer ( $\mathrm{pH}$ 8.43) was used to ensure deprotonation of the carboxylic groups on the surface of GQDs to enable electrophoretic mobility under an electrical field [52]. As shown in Fig. 5a, the commercially available GQD mixture was shown to contain three prominent fluorescence bands after 
electrophoresis. The bands were separated using MP-PAGE based on tracking of their visible fluorescence. As shown in Fig. 5b, the stock sample shows the maximum fluorescence peak at about 535 $\mathrm{nm}$, which is in the range of the peaks observed from the isolated bands at $524,549 \mathrm{~nm}$, and $575 \mathrm{~nm}$ for bands 1, 2, and 3, respectively. A comparison with Fig. 5a reveals that the bands show an overall redshifting of their fluorescence emissions with decreasing mobilities. These results are in agreement with literature, which predicts red-shifting of the emission fluorescence with increasing size $[45,53,54]$, thus indicating MP-PAGE was able to separate different GQD sizes in each band. We note, however, an overall stronger signal for bands 1 and 2 in the gel (Fig. $5 a$ ) as well as a closer proximity of the mixture's peak fluorescence to those of bands 1 and 2 compared to that of band 3 (Fig. $5 \mathrm{~b}$ ). Although such findings indicate higher concentrations of GQDs from bands 1 and 2 in the commercial mixture, differences in the quantum yields of the three bands at the given excitation wavelength may also lead to similar observations.

In addition to the fluorescence characterizations, the bands were further analyzed using UV-visible absorption spectroscopy. As shown in Fig. 5c, the three bands have distinct absorption spectra. In particular, band 3 shows the narrowest absorption features, with a peak absorption at $229 \mathrm{~nm}$, whereas band 1 shows the broadest absorption range, including additional peaks at 268,460 , and $488 \mathrm{~nm}$, the latter of which is in closest resonance with the $485 \mathrm{~nm}$ excitation used in the fluorescence analysis. Although the commercial mixture contains the same overlapping peaks as those observed in the three separation bands, it also contains additional peaks, for example at $365 \mathrm{~nm}$, as well as sharper peaks at 277 and $325 \mathrm{~nm}$. The presence of additional absorption peaks in the mixture that are not captured by the separated bands could be indicative of absorbing moieties that are non-fluorescent under the conditions used to track and separate the bands. Nonetheless, the distinct absorption spectra observed from these three bands confirm the fractionation of GQDs with different optoelectronic properties.

\section{Outlook And Conclusions}

Numerous studies have reported the use of gel electrophoresis for the preparative purification of proteins either in column or slab formats $[16,18,21,55,56]$. These studies relied on either continuous or stoppedflow elution during electrophoresis or methods that require gel slicing after electrophoresis, followed by subsequent extraction steps that limit the separation yield [25]. Although a variety of such apparatuses have become available commercially, they are complex, expensive, or difficult to set up [17]. The MPPAGE, presented herein, utilizes standard electrophoretic equipment readily available in most bioanalysis laboratories for purifying different biological samples including proteins, nucleic acids, and bioconjugates. Unlike other preparative gel electrophoresis methods that require high elution volumes and yield diluted protein extracts, MP-PAGE samples are eluted in a small volume (1-2 $\mathrm{ml}$ ) of buffer that can be collected with a syringe. The MP-PAGE setup also circumvents the need for not only complex gel preparation and shutdown procedures but also specialized equipment such as elution chambers, external pumps, and external fraction collectors. 
The versatility of new MP-PAGE method was demonstrated by purifying four distinct samples: a nucleic acid, a protein, a bioconjugate, and a synthetic nanoparticle sample in multicomponent mixtures. The bioconjugate sample used in this study could not be purified using existing techniques, particularly due to the low sample amount. Although all the samples used in this work were (or labeled to become) fluorescent to allow us to visually track the samples in the gel, the technique is applicable to nonfluorescent samples as well. For example, protein samples could be pre-stained with dyes such as Coomassie G-250 [17] and Instant Band (EZBiolab) and run in parallel to the MP-PAGE to track the position of protein bands and identify the appropriate collection time for the protein.

The MP-PAGE method is convenient for fast and cost-effective, small-volume protein purification in research laboratories. The protein product purity achieved with MP-PAGE is comparable to the purity obtained using chromatography techniques, which are considered as the gold-standard for protein purification. In this study, MP-PAGE purification was performed within $2 \mathrm{~h}$ compared to the two-step IMAC+SEC purification that required nearly one day to achieve comparable purities. The ability to tailor the separation resolution by altering the resolving gel concentration further provides a low-cost, tunable approach for purifying proteins of various sizes and charges. In contrast, chromatography techniques require different columns for proteins of different sizes, charges, or metal ion affinities. Compared to IMAC, MP-PAGE also does not require a protein tag for purification, circumventing the need for further protein engineering and overcoming losses from subsequent de-tagging steps. Furthermore, previous studies have shown that native PAGE can be used to separate and purify proteins from intact $E$. coli without disrupting the cells [21]. This ability would allow MP-PAGE to separate proteins in the absence of preliminary cell disruption steps that are necessary for chromatography techniques and can contribute to additional protein loss. We note, however, that these advantages are largely limited to applications that require limited protein amounts. In such cases, chromatography and alternative flow-through systems remain beneficial for larger volume protein purification.

MP-PAGE can also be extended to separate other biomolecules, such as DNA, DNA-protein conjugates, and nanoparticles. The MP-PAGE separation demonstrated in this study, for example, was performed under native conditions based on differences in both protein charge and size. MP-PAGE can also be performed in SDS-denaturing conditions, which can separate proteins only based on size. However, though SDS separation benefits from improved separation resolution compared to continuous nativePAGE, this application would be limited to proteins that can be refolded. In addition, this platform can also be applied to specialized biomolecules such as oligonucleotide origami and nanoparticle conjugates. As is the case with proteins and DNA, separation of these conjugates can be achieved by varying concentration, height, and thickness of the resolving gel, as well as buffer composition and applied potential in the electrophoresis device. The use of alternative gel systems, such as discontinues $\mathrm{pH}$ gradient or PAG concentration gradient gels, could also be implemented to further tune and optimize separation.

\section{Abbreviations}


Micro-preparative polyacrylamide gel electrophoresis: MP-PAGE; graphene quantum dots : GQDs ; enhanced yellow fluorescent protein : EYFP; size exclusion chromatography: SEC: immobilized metal-ion affinity chromatography: IMAC.

\section{Declarations}

\section{Availability of data and materials}

All data are available upon request.

\section{Competing interests}

There are no conflicts to declare.

\section{Funding}

This work was supported by the Swiss National Science Foundation (Assistant Professor (AP) Energy Grant) and the Ferdowsi University of Mashhad (Grant No. 3/ 42787).

\section{Authors' contributions}

SHS designed and completed the experiments. SHS prepared protein and bioconjugates with help of SJW and VZ. SHS and SJW wrote the first draft. EKG participated in discussion. AAB and HA supervised the projects. $A A B$ edited the final version of the manuscript and all the authors reviewed and approved the final manuscript.

\section{References}

1. Ersson B, Rydén L, Janson JC (2011) Introduction to Protein Purification. In: Protein Purification: Principles, High Resolution Methods, and Applications: Third Edition. John Wiley \& Sons, pp 1-22

2. Cupido T, Pisa R, Kelley ME, Kapoor TM (2019) Designing a chemical inhibitor for the AAA protein spastin using active site mutations. Nat Chem Biol 15:444-452. https://doi.org/10.1038/s41589-0190225-6

3. Guyomar C, Thépaut M, Nonin-Lecomte S, et al (2020) Reassembling green fluorescent protein for in vitro evaluation of trans-translation. Nucleic Acids Res 48:E22. https://doi.org/10.1093/nar/gkz1204

4. Nickerson JL, Doucette AA (2020) Rapid and Quantitative Protein Precipitation for Proteome Analysis by Mass Spectrometry. J Proteome Res 19:2035-2042. https://doi.org/10.1021/acs.jproteome.9b00867

5. Foret F, Datinská V, Voráčová I, et al (2019) Macrofluidic Device for Preparative Concentration Based on Epitachophoresis. Anal Chem 91:7047-7053. https://doi.org/10.1021/acs.analchem.8b05860 
6. Goldring JPD (2019) Concentrating proteins by salt, polyethylene glycol, solvent, sds precipitation, three-phase partitioning, dialysis, centrifugation, ultrafiltration, lyophilization, affinity chromatography, immunoprecipitation or increased temperature for protein isolati. In: Methods in Molecular Biology. Humana Press Inc., pp 41-59

7. Tsushima M, Sato S, Nakamura H (2017) Selective purification and chemical labeling of a target protein on ruthenium photocatalyst-functionalized affinity beads. Chem Commun 53:4838-4841. https://doi.org/10.1039/C7CC01595J

8. Yamamichi S, Jinno Y, Haraya N, et al (2011) Separation of proteins using supramolecular gel electrophoresis. Chem Commun 47:10344-10346. https://doi.org/10.1039/C1CC13826J

9. Fekete S, Guillarme D (2014) Ultra-high-performance liquid chromatography for the characterization of therapeutic proteins. TrAC - Trends Anal Chem 63:76-84. https://doi.org/10.1016/j.trac.2014.05.012

10. Carta G, Jungbauer A (2020) Introduction to Protein Chromatography. In: Protein Chromatography: Process Development and Scale-Up. John Wiley \& Sons, pp 63-91

11. Sajjadi SH, Goharshadi EK, Ahmadzadeh H (2019) Heat dissipation in slab gel electrophoresis: The effect of embedded TiO 2 nanoparticles on the thermal profiles. J Chromatogr B Anal Technol Biomed Life Sci 1118-1119:63-69. https://doi.org/10.1016/j.jchromb.2019.04.030

12. Sajjadi SH, Ahmadzadeh H, Goharshadi EK (2020) Enhanced electrophoretic separation of proteins by tethered SiO2 nanoparticles in an SDS-polyacrylamide gel network. Analyst 145:415-423. https://doi.org/10.1039/c9an01759c

13. Wu SJ, Schuergers N, Lin KH, et al (2018) Restriction Enzyme Analysis of Double-Stranded DNA on Pristine Single-Walled Carbon Nanotubes. ACS Appl Mater Interfaces 10:37386-37395. https://doi.org/10.1021/acsami.8b12287

14. Westermeier R, Gronau S, Becket P, et al (2005) Electrophoresis in Practice: A Guide to Methods and Applications of DNA and Protein Separations: Fourth Edition. John Wiley \& Sons

15. Sun SM, Hall TC (1974) Application of remazol dye for isolation of protein subunits by preparative SDS-polyacrylamide gel electrophoresis. Anal Biochem 61:237-242. https://doi.org/10.1016/00032697(74)90350-9

16. Naryzhny SN (1996) Upside-down stopped-flow electrofractionation of complex protein mixtures. Anal Biochem 238:50-53. https://doi.org/10.1006/abio.1996.0249

17. Jian-Hua ZXGC, Lu-Yin Y, Li T, et al (2012) Separation and isolation of fusion protein using a new native preparative PAGE device. J Chromatogr Sci 50:820-825.

https://doi.org/10.1093/chromsci/bms077 
18. Hao F, Li J, Zhai R, et al (2016) A novel microscale preparative gel electrophoresis system. Analyst 141:4953-4960. https://doi.org/10.1039/c6an00780e

19. Groschup MH, Boschwitz J, Timoney JF (1991) A convenient gel holder for preparative electrophoretic separation of aggregated bacterial proteins. Electrophoresis 12:90-93. https://doi.org/10.1002/elps.1150120117

20. Fadouloglou VE (2013) Electroelution of nucleic acids from polyacrylamide gels: A custom-made, agarose-based electroeluter. Anal Biochem 437:49-51. https://doi.org/10.1016/j.ab.2013.02.021

21. Chew FN, Tan WS, Ling TC, Tey BT (2011) Optimization of a native gel electrophoretic process for the purification of intracellular green fluorescent protein from intact Escherichia coli cells. Process Biochem 46:399-403. https://doi.org/10.1016/j.procbio.2010.07.032

22. Carpenter HC, Skerritt JH, Wrigley CW, Margolis J (1986) A device for preparative elution electrophoresis using a polyacrylamide gel slab. Electrophoresis 7:221-226. https://doi.org/10.1002/elps.1150070507

23. Shain DH, Yoo J, Slaughter RG, et al (1992) Electrofractionation: A technique for detecting and recovering biomolecules. Anal Biochem 200:47-51. https://doi.org/10.1016/0003-2697(92)90275-C

24. Krause RGE, Dean Goldring JP (2019) Continuous Elution SDS-PAGE with a Modified Standard Gel Apparatus to Separate and Isolate an Array of Proteins from Complex Mixtures BT - Electrophoretic Separation of Proteins: Methods and Protocols. In: Kurien BT, Scofield RH (eds). Springer New York, New York, NY, pp 467-478

25. Seelert $H$, Krause $F$ (2008) Preparative isolation of protein complexes and other bioparticles by elution from polyacrylamide gels. Electrophoresis 29:2617-2636.

https://doi.org/10.1002/elps.200800061

26. Rosen CB, Kodal ALB, Nielsen JS, et al (2014) Template-directed covalent conjugation of DNA to native antibodies, transferrin and other metal-binding proteins. Nat Chem 6:804-809. https://doi.org/10.1038/nchem.2003

27. O'Meara TR, O'Meara MJ, Polvi EJ, et al (2019) Global proteomic analyses define an environmentally contingent Hsp90 interactome and reveal chaperone-dependent regulation of stress granule proteins and the R2TP complex in a fungal pathogen. PLOS Biol 17:e3000358

28. Zhou Z, Xiang Y, Tong A, Lu Y (2014) Simple and efficient method to purify DNA-protein conjugates and its sensing applications. Anal Chem 86:3869-3875. https://doi.org/10.1021/ac4040554

29. Yang L, Bui L, Hanjaya-Putra D, Bruening ML (2020) Membrane-Based Affinity Purification to Identify Target Proteins of a Small-Molecule Drug. Anal Chem 92:11912-11920. https://doi.org/10.1021/acs.analchem.0c02316 
30. Zubkovs V, Wu SJ, Rahnamaee SY, et al (2020) Site-specific protein conjugation onto fluorescent single-walled carbon nanotubes. Chem Mater 32:8798-8807. https://doi.org/10.1021/acs.chemmater.0c02051

31. Maxam AM, Gilbert W (1977) A new method for sequencing DNA. Proc Natl Acad Sci U S A 74:560564. https://doi.org/10.1073/pnas.74.2.560

32. De Meulenaere E, Nguyen Bich N, De Wergifosse M, et al (2013) Improving the second-order nonlinear optical response of fluorescent proteins: The symmetry argument. J Am Chem Soc 135:4061-4069. https://doi.org/10.1021/ja400098b

33. Green MR, Sambrook J (2019) Isolation of DNA fragments from polyacrylamide gels by the crush and soak method. Cold Spring Harb Protoc 2019:143-146. https://doi.org/10.1101/pdb.prot100479

34. Snapp EL, McCaul N, Quandte M, et al (2017) Structure and topology around the cleavage site regulate post-translational cleavage of the HIV-1 gp160 signal peptide. Elife 6:e26067. https://doi.org/10.7554/eLife.26067

35. Saccà B, Niemeyer CM (2011) Functionalization of DNA nanostructures with proteins. Chem Soc Rev 40:5910-5921. https://doi.org/10.1039/c1cs15212b

36. Hermanson GT (2013) Introduction to Bioconjugation. In: Hermanson GTBT-BT (Third E (ed) Bioconjugate Techniques, 3rd ed. Academic Press, Boston, pp 1-125

37. Singh Y, Murat P, Defrancq E (2010) Recent developments in oligonucleotide conjugation. Chem Soc Rev 39:2054-2070. https://doi.org/10.1039/b911431a

38. Yan X, Zhang H, Wang Z, et al (2018) Quantitative synthesis of protein-DNA conjugates with 1:1 stoichiometry. Chem Commun 54:7491-7494. https://doi.org/10.1039/C8CC03268H

39. Cui C, Zhang H, Wang R, et al (2017) Recognition-then-Reaction Enables Site-Selective Bioconjugation to Proteins on Live-Cell Surfaces. Angew Chemie Int Ed 56:11954-11957. https://doi.org/https://doi.org/10.1002/anie.201706285

40. Yang YR, Liu Y, Yan H (2015) DNA Nanostructures as Programmable Biomolecular Scaffolds. Bioconjug Chem 26:1381-1395. https://doi.org/10.1021/acs.bioconjchem.5b00194

41. Khatwani SL, Kang JS, Mullen DG, et al (2012) Covalent protein-oligonucleotide conjugates by copper-free click reaction. Bioorganic Med Chem 20:4532-4539. https://doi.org/10.1016/j.bmc.2012.05.017

42. Lapiene V, Kukolka F, Kiko K, et al (2010) Conjugation of fluorescent proteins with DNA oligonucleotides. Bioconjug Chem 21:921-927. https://doi.org/10.1021/bc900471q 
43. Pan D, Guo L, Zhang J, et al (2012) Cutting sp2clusters in graphene sheets into colloidal graphene quantum dots with strong green fluorescence. J Mater Chem 22:3314-3318. https://doi.org/10.1039/C2JM16005F

44. Patel KD, Singh RK, Kim H-W (2019) Carbon-based nanomaterials as an emerging platform for theranostics. Mater Horizons 6:434-469. https://doi.org/10.1039/C8MH00966J

45. Sk MA, Ananthanarayanan A, Huang L, et al (2014) Revealing the tunable photoluminescence properties of graphene quantum dots. J Mater Chem C 2:6954-6960.

https://doi.org/10.1039/C4TC01191K

46. Chung S, Revia RA, Zhang M (2021) Graphene Quantum Dots and Their Applications in Bioimaging, Biosensing, and Therapy. Adv Mater 33:1904362.

https://doi.org/https://doi.org/10.1002/adma.201904362

47. Yan Y, Gong J, Chen J, et al (2019) Recent Advances on Graphene Quantum Dots: From Chemistry and Physics to Applications. Adv Mater 31:1808283.

https://doi.org/https://doi.org/10.1002/adma.201808283

48. Sajjadizadeh H-S, Goharshadi EK, Ahmadzadeh H (2020) Photoelectrochemical water splitting by engineered multilayer Ti02/GQDs photoanode with cascade charge transfer structure. Int J Hydrogen Energy 45:123-134. https://doi.org/https://doi.org/10.1016/j.ijhydene.2019.10.161

49. Hasan MT, Gonzalez-Rodriguez R, Ryan C, et al (2019) Variation of Optical Properties of Nitrogendoped Graphene Quantum Dots with Short/Mid/Long-wave Ultraviolet for the Development of the UV Photodetector. ACS Appl Mater Interfaces 11:39035-39045. https://doi.org/10.1021/acsami.9b10365

50. Ouyang Z, Lei Y, Chen Y, et al (2019) Preparation and Specific Capacitance Properties of Sulfur, Nitrogen Co-Doped Graphene Quantum Dots. Nanoscale Res Lett 14:219.

https://doi.org/10.1186/s11671-019-3045-4

51. Liu D, Li HJ, Gao J, et al (2018) High-Performance Ultraviolet Photodetector Based on Graphene Quantum Dots Decorated ZnO Nanorods/GaN Film Isotype Heterojunctions. Nanoscale Res Lett 13:261. https://doi.org/10.1186/s11671-018-2672-5

52. Davardoostmanesh M, Goharshadi EK, Ahmadzadeh H (2019) Electrophoretic size fractionation of graphene oxide nanosheets. New J Chem 43:5047-5054. https://doi.org/10.1039/C8NJ06411C

53. Bailey RE, Nie S (2003) Alloyed Semiconductor Quantum Dots: Tuning the Optical Properties without Changing the Particle Size. J Am Chem Soc 125:7100-7106. https://doi.org/10.1021/ja035000o

54. Cheng H-M, Lin K-F, Hsu H-C, Hsieh W-F (2006) Size dependence of photoluminescence and resonant Raman scattering from ZnO quantum dots. Appl Phys Lett 88:261909.

https://doi.org/10.1063/1.2217925

Page 16/22 
55. Laremore TN, Ly M, Solakyildirim K, et al (2010) High-resolution preparative separation of glycosaminoglycan oligosaccharides by polyacrylamide gel electrophoresis. Anal Biochem 401:236-241. https://doi.org/10.1016/j.ab.2010.03.004

56. Gabe CM, Brookes SJ, Kirkham J (2017) Preparative SDS PAGE as an alternative to his-tag purification of recombinant Amelogenin. Front Physiol 8:424. https://doi.org/10.3389/fphys.2017.00424

\section{Figures}
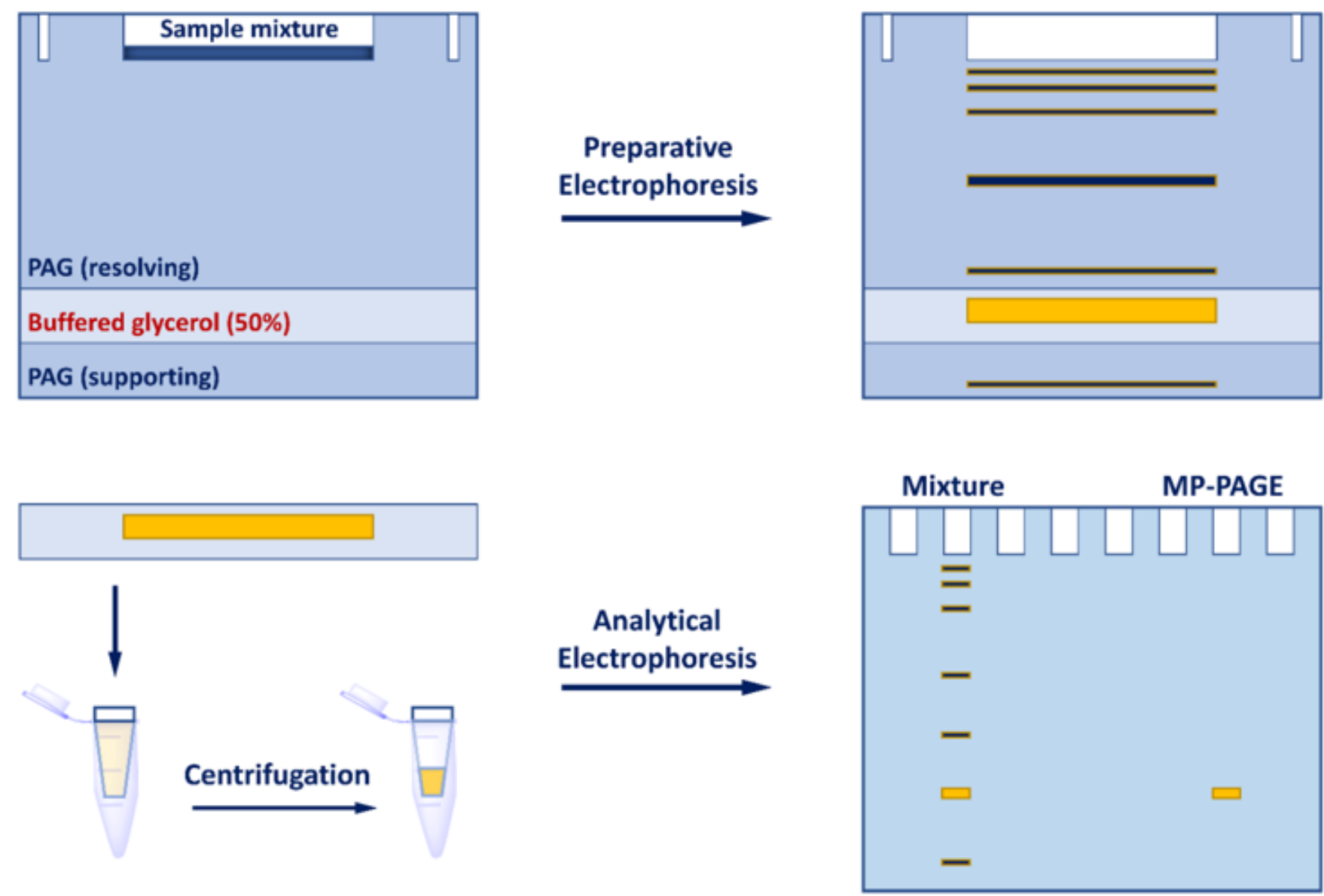

\section{Figure 1}

A schematic drawing of MP-PAGE for separating and extracting nanoscale materials. The upper-left schematic shows the composition of a MP-PAGE gel containing a top resolving PAG, a glycerol layer for sample extraction, and a bottom supporting gel. The resolving gel is used at an adequate gel composition and run at appropriate voltages for biomolecule/nanoparticle separation. The band containing the desired biomolecule of interest (yellow band, top-right) is selectively eluted into the glycerol layer and run through a centrifugal filter (bottom-left) to remove the glycerol. An analytical gel is run on the extract (bottom-right) to confirm sample purity relative to the initial mixture. 
A

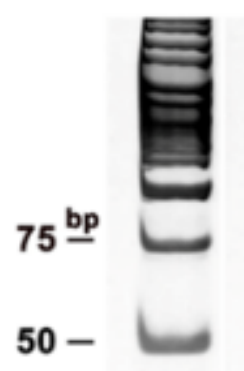

$25-$

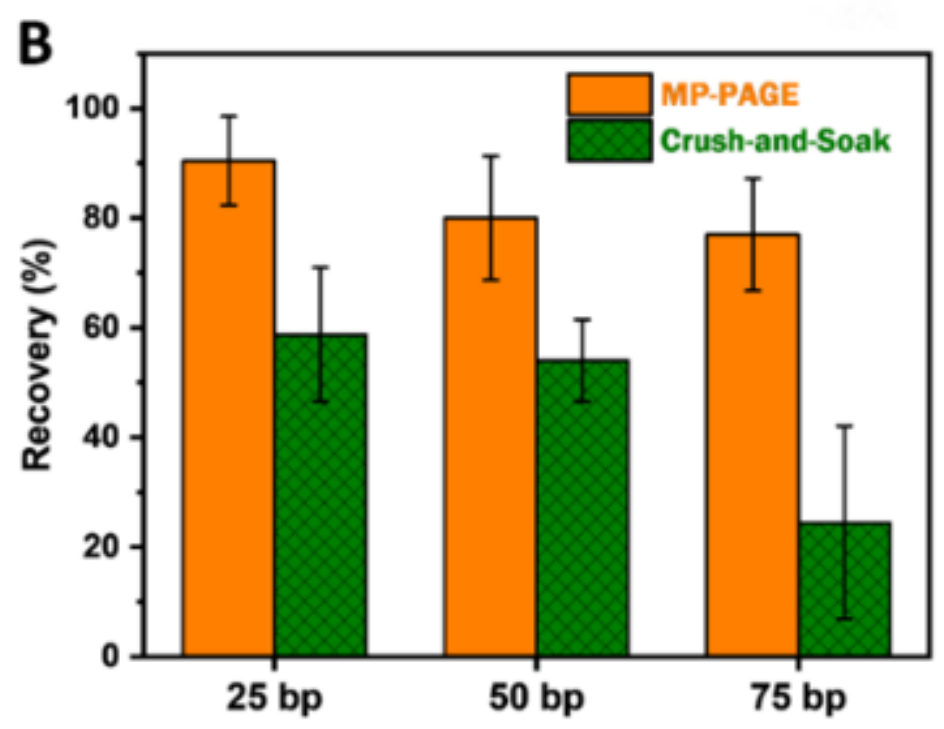

Figure 2

Separation of ds-DNA lengths from a ladder mixture containing 12 different DNA lengths. (A) $12 \%$ Native PAGE profiles (stained with SG) of the MP-PAGE-separated DNA samples. (B) Comparison of the purified DNA recovery yields from the MP-PAGE and the Crush-and-Soak techniques. Experiments were performed in triplicates, and the error bars represent standard deviations. 
A

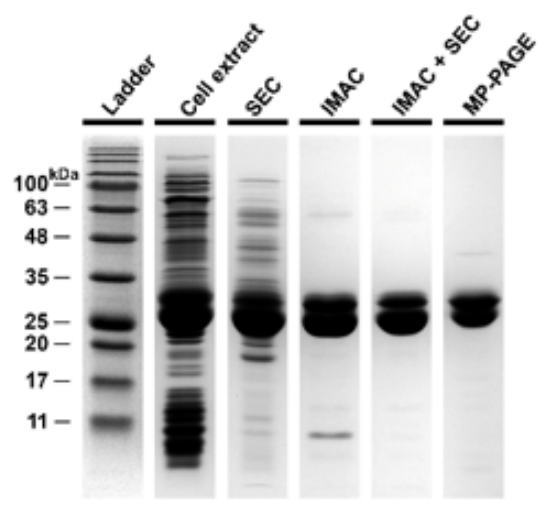

B

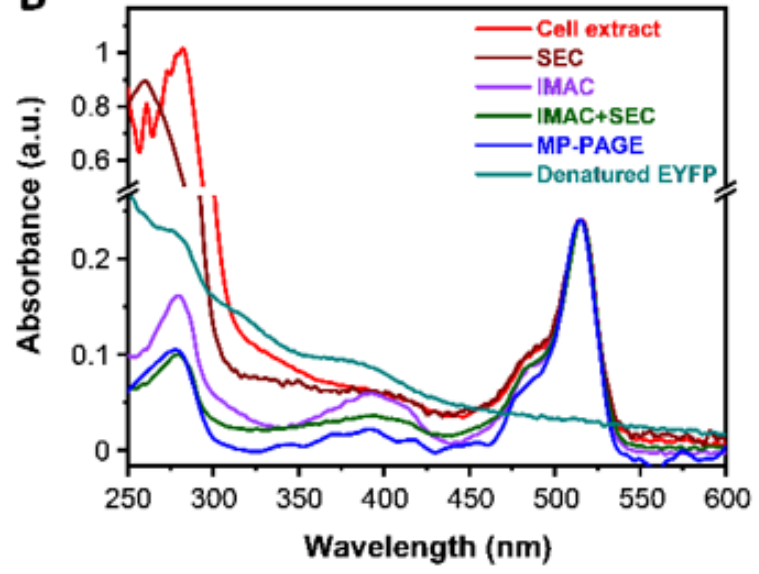

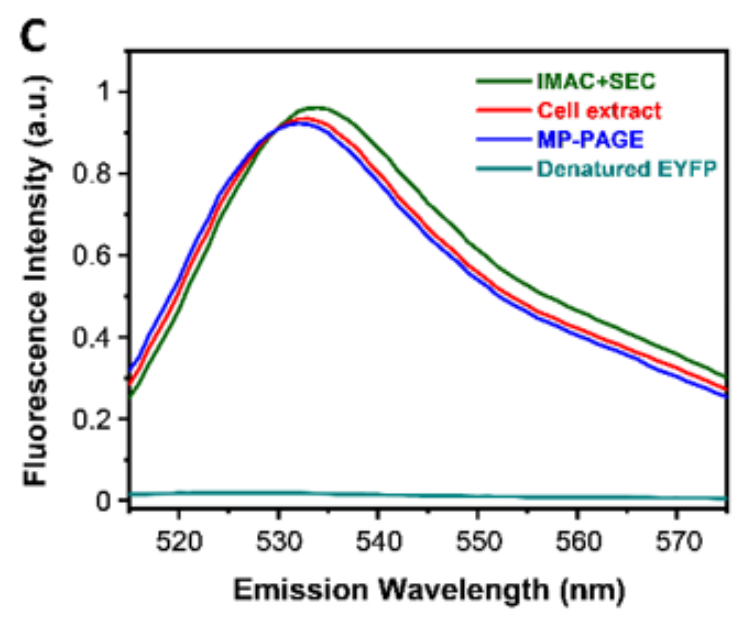

\section{Figure 3}

Analysis of protein-containing samples using gel electrophoresis, absorbance, and fluorescence spectroscopies. (A) CB-stained reducing SDS-PAGE profiles of crude cell extract and purified EYFP samples from SEC, IMAC, IMAC + SEC, and MP-PAGE. To compare the amount of impurities for an equivalent amount of EYFP, each lane was loaded with sample volumes that have the same EYFP peak absorption at $514 \mathrm{~nm}(\mathrm{~A} 514 \mathrm{~nm}=0.2)$. (B) UV-visible spectra of crude cell extract, purified EYFP samples achieved by SEC, IMAC, IMAC + SEC, and MP-PAGE, as well as denatured EYFP. Denatured EYFP corresponds to the IMAC+SEC sample heated to $95 \mathrm{oC}$ for $5 \mathrm{~min}$. (C) Fluorescence spectra of crude cell extract and purified EYFP samples from IMAC+SEC (as the purest protein sample) and MP-PAGE, as well as denatured EYFP described in (B). The samples were excited at $490 \mathrm{~nm}$ and fluorescence spectra were normalized by the EYFP concentration. 


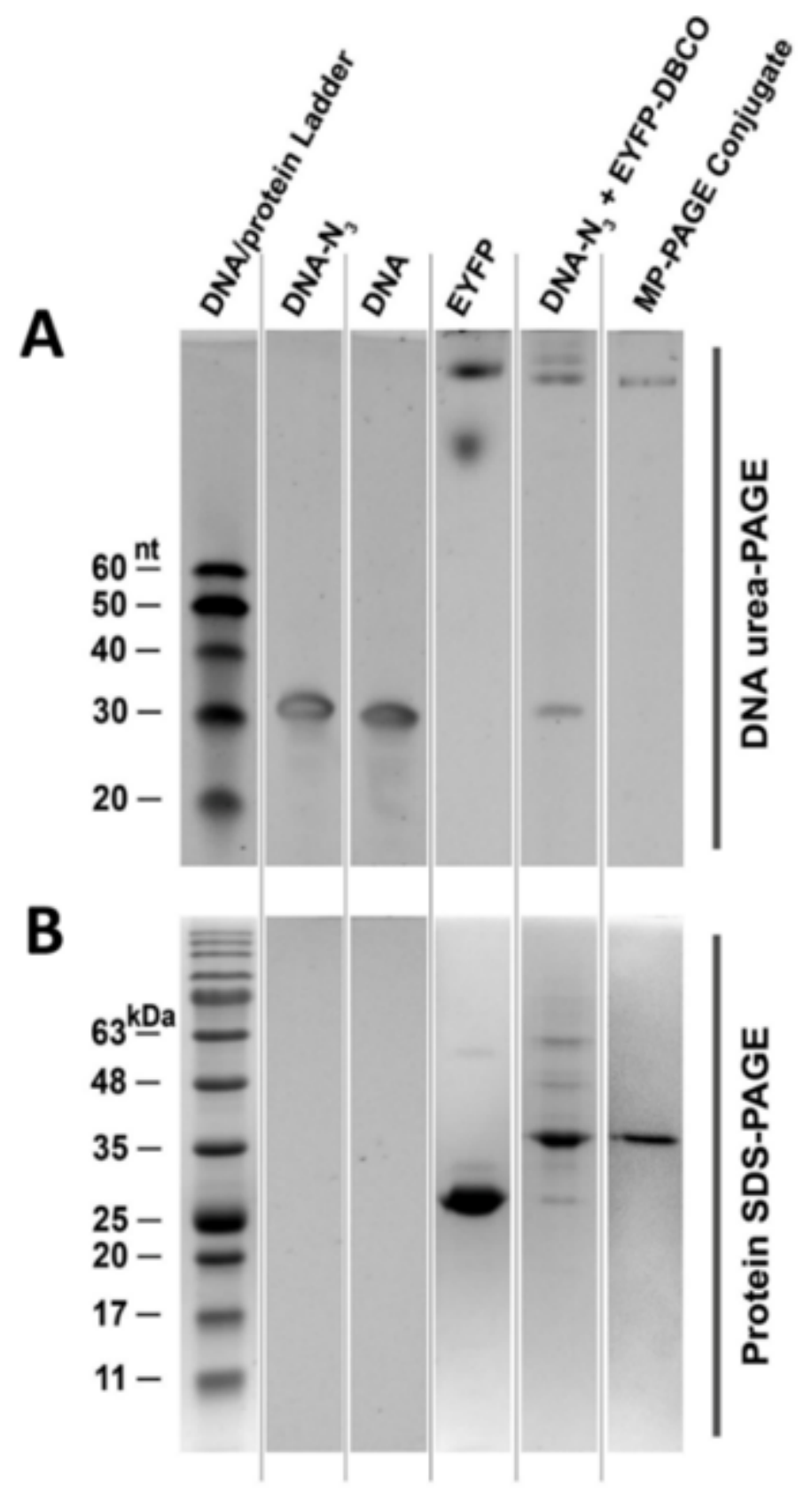

Figure 4

Denaturing PAGE analysis of ssDNA-EYFP conjugate samples. (A) Urea-PAGE profiles showing DNA, EYFP, and conjugate bands. (B) Reducing SDS-PAGE profiles showing EYFP and conjugate bands. DNA$\mathrm{N} 3+$ EYFP-DBCO corresponds to the reaction sample after $4 \mathrm{~h}$ incubation followed by filtration using a 30 $\mathrm{kDa}$ Amicon centrifugal device. 
A

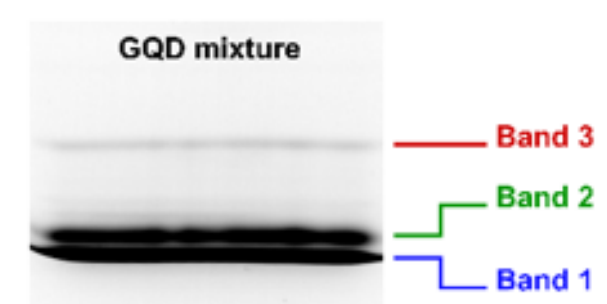

B
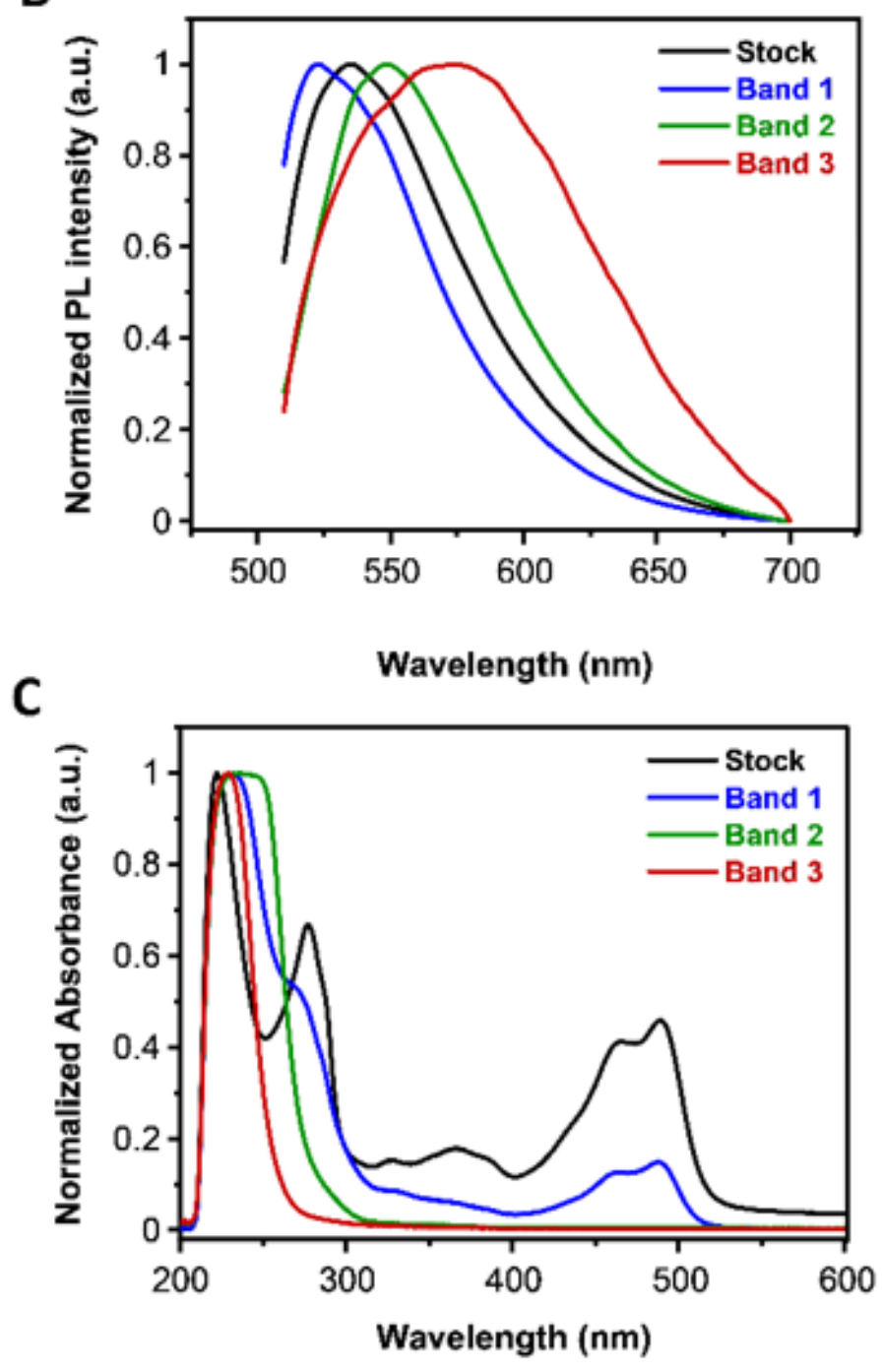

\section{Figure 5}

Separation of GQDs of different sizes. (A) PAGE profile of commercial GQD sample. The separated bands are visible under blue light. (B) fluorescence spectra $\left(\lambda \_e x=485 \mathrm{~nm}\right.$ ) and $(C)$ UV-visible spectra of the stock solution and the separated fractions shown in $(A)$.

\section{Supplementary Files}


This is a list of supplementary files associated with this preprint. Click to download.

- MPPAGEsupp.docx 